\title{
Interactive Virtual Angioscopy
}

\author{
Enrico Gobbetti* $\quad$ Piero Pili ${ }^{\dagger} \quad$ Antonio Zorcolo ${ }^{\ddagger} \quad$ Massimiliano Tuveri§ M.D. \\ CRS4 \\ Center for Advanced Studies, Research and Development in Sardinia \\ Cagliari, Italy $₫$
}

\begin{abstract}
Virtual angioscopy is a non invasive medical procedure for exploring parts of the human vascular system. We have developed an interactive tool that takes as input data acquired with standard medical imaging modalities and regards it as a virtual environment to be interactively inspected. The system supports real-time navigation with stereoscopic direct volume rendering and dynamic endoscopic camera control, interactive tissue classification, and interactive point picking for morphological feature measurement. In this paper, we provide an overview of the system, discuss the techniques used in our prototype, and present experimental results on human data sets. The accompanying video-tape illustrates our approach with interactive sequences showing the examination of a human carotid artery.
\end{abstract}

Keywords: Virtual angioscopy, endoscopy, interactive rendering, volume rendering, virtual environment

\section{Motivation}

Atherosclerosis is a generalized process in which morbidity and mortality usually result from localized plaque deposition rather than diffuse diseases. The locations of the depositions in susceptible zones of the arterial tree are relatively constant and predictable, which suggests that vessel geometry and fluid localizing factors play an important role in the onset of the disease. Differing lumen diameters, curvatures, branching, and angles produce local disturbance in the primary blood flow field. Branch points are known to be particularly vulnerable to plaque formation and are subject to wide variation in hemodynamic conditions. Emerging approaches to vascular disease treatment are percutaneous transluminal angioplasty and/or intravascular stenting after angioplasty. The effectiveness of these techniques remains however limited by a $30 \%$ to $50 \%$ restenosis rate within the first six months. One of the reasons of this high failure rate is the difficulty to characterize the arterial lesion, the precise three-dimensional geometry of the atherosclerotic

\footnotetext{
*Enrico.Gobbetti@crs4.it

†Piero.Pili@crs4.it

‡Antonio.Zorcolo@crs4.it

§Massimiliano.Tuveri@crs4.it

ฯCRS4, Via N. Sauro 10, 09123 Cagliari, Italy, http://www.crs4.it
}

plaque, and its relationship with the rest of the vessel. Technological advances and improvements in the characterization of the diseased vessel as well as in the understanding of the precise spatial location of the lesion to be stented are susceptible to increase the success rate of vascular procedures and the feasibility of approaching complex lesions, thus reducing the rate of restenosis.

Recent advances in non-invasive data acquisition technology, e.g. spiral computerized tomography $(\mathrm{CT})$ and fast magnetic resonance (MR) imaging, are now providing physicians with large amounts of quantitative information on a patient's internal structures, making it possible to carefully study parts of the arterial tree before intervention. The ability to analyze scalar volume datasets acquired by the use of non invasive techniques is thus gaining importance in the diagnostic and therapy planning fields, and physicians are now faced with the need to interpret data produced by $\mathrm{CT}$ and MR scanners.

Data analysis is currently done mostly by analyzing static images of 2D volume slices. Physicians are thus forced to mentally combine multiple views for constructing a mental model of 3D shapes, adding further complexity to what is often an already difficult task. The creation of a virtual environment for the direct analysis in three dimensions of volumetric data, i.e. a virtual angioscopy system, overcomes these problems $[12,7]$.

In virtual angioscopy, and more generally virtual endoscopy, a three-dimensional dataset of a body part derived from diagnostic imaging data is displayed on a computer and made available for $3 \mathrm{D}$ navigation and analysis. Research virtual endoscopy prototypes are being developed and have proven useful for a variety of clinical applications (e.g. bronchoscopy [23], cholonoscopy [10] and minimally invasive surgery planning [1]).

Because of the computational costs associated with image production, work in this field has until recently concentrated on generating precomputed fly-through animations [12, 19, 22], which makes these tools rather cumbersome to be used for the kind of detailed clinical examinations that are needed to examine complex vessel lesions. The continued improvement and proliferation of graphics hardware for workstations and personal computers promises to bring increasing prominence to a newer style of virtual endoscopy system, which support real-time exploration and are therefore better suited for virtual angioscopy applications. However, current interactive virtual endoscopy systems are mostly based on surface rendering techniques, where volumetric data is automatically or manually segmented during a preprocessing step to extract boundary surfaces that are then rendered during navigation $[23,10]$. These techniques have proven to be often not accurate enough for clinical examinations $[29,16,7]$. In particular, since when using surface rendering only the interior surfaces of a cavity are visible during navigation, diagnostic capabilities are severely limited $[17,18,22]$.

Our aim is to merge the concepts of interactive direct volume rendering and virtual endoscopy in a tool for non-invasive visualization and analysis of diseased vessels. In this paper, we provide an overview of the system, discuss the techniques used in our prototype, and present experimental results on explanted human data 
sets. The accompanying video-tape illustrates our approach with interactive sequences showing the examination of human carotid artery CT data.

\section{System Overview}

Our interactive virtual angioscopy system takes as input data acquired with standard medical imaging modalities and regards it as a virtual environment to be interactively inspected. As the system employs the DICOM standard for input dataset coding, it is possible to have it connected directly to standard medical data acquisition devices.

Our laboratory is connected via LAN through a $2 \mathrm{Mbit} / \mathrm{s}$ dedicated line to a CTN Image Server [28] that can acquire datasets from a Picker CT Scanner equipped with a DICOM Storage Class Provider located in the Radiology Department of the S. Michele hospital [8]. New acquisitions are made thus available for examination in a few minutes. When the dataset is loaded on the graphical workstation, the system presents a perspective view of the dataset and users may interactively take morphological measures, move the viewpoint, and modify the classification parameters to obtain the best visual representation of the particular anatomy under examination.

When building a virtual angioscopy system, the most important design factors to be taken into account are rendering and interaction techniques. Our choices are discussed in the following sections.

\subsection{Rendering Techniques}

Direct volume visualization is a rendering technique which does not require the construction of intermediate geometric representations and has the advantage of supporting the representation in a single image of the full dataset using semi-transparent mapping [20, 26, 6].

With this technique, images are produced by integrating along selected projectors the value of a continuous absorption-emission volume function reconstructed from discrete sampling points [13]. By manipulating the mapping from values of the original volume data to emission and absorption coefficients, various effects can be achieved, including isosurfaces and opaque objects. The method is therefore more general than surface rendering and more appropriate for tissue examination.

The full potential of volume rendering is exploited when allowing users to view and interact in real-time with the volume in a natural way. To support interactive performance, we have chosen to directly render the volumes via back-to-front volume slices composition, exploiting texture mapping and alpha-blending on high-end graphics accelerators [15]. While not as general as ray-casting, this type of rendering technique has proven to be appropriate for medical data exploration [4, 3, 27].

Our implementation, based on OpenGL 3D texturing, supports perspective projection with arbitrary viewing angles, 16 bit texels, arbitrarily sized volumes through brick decomposition, and interactive classification through hardware look-up tables. Several recent papers describe similar uses of 3D texture mapping hardware for direct volume rendering $[4,3,9,27,11]$. Our major improvements are the following:

- adaptive sampling: both image quality and rendering speed are directly related to the number of slices used to sample the volume. The more slices are rendered, the more accurate the results, but the longer the rendering time. Given the timing constraints of interactive applications, it is important to produce good quality images without using an excessively large number of slices. Since in perspective renderings the contribution of the slices to the final image diminishes with their distance to the viewer, a good strategy is to space the slices proportionally to the inverse of that distance. With this approach spatial resolution is increased close to the viewer, making close-up inspection more precise while reducing the details in the background, which is appropriate for an interactive tool. As emission/absorption varies non linearly with thickness [13], special care has to be taken to correctly compute the contribution of each slice. In our renderer, we associate to each slice a thickness which depends on slice spacing. Texture lookup tables, a Silicon Graphics extension to OpenGL, are then used to define opacity values that depend on slice thickness. Serra et al. also use variable slice spacing, but without opacity correction, introducing notable artifacts [21];

- zoom rendering: pixel fill-rate is the major limiting factor when using a texturing approach to volume visualization. In zoom rendering, an appropriately down-scaled image is rendered in the back buffer and then enlarged and copied to the front buffer [14]. This way, delays associated with buffer swap synchronization are avoided, and the number of pixels filled during volume rendering is reduced. In our implementation, the copy and zoom operations are implemented by copying the reduced size image in texture memory and then rendering a textured polygon in the front buffer. This way, sophisticated texture interpolation algorithms can be used to reduce the artifacts caused by magnification. Zoom rendering is particularly useful for endoscopic volume rendering applications, because the pixel resolution is much larger than the resolution of the data that is displayed in the window. Zoom rendering is only active during navigation, still images being recomputed at maximum resolution.

The ability to render perspective views of a volume at interactive speeds dramatically improves depth perception, thanks to motion parallax effects, and makes it possible to use direct interaction techniques to naturally perform complex $3 \mathrm{D}$ tasks, such as finding the best viewpoint or positioning cutting planes. Depth shading and stereoscopic image display provide additional depth cues, helping in the perception of $3 \mathrm{D}$ shapes. To provide accurate visualization beyond the capabilities of a real-time volume renderer, we integrated in our virtual angioscopy prototype a volume raycaster, available as a viewing filter [2] that produces on demand a ray-casted view of a user selectable $2 \mathrm{D}$ screen region.

\subsection{Interaction Techniques}

In our application, the user interacts with the rendered volume using both hands simultaneously: the left hand, controlling a Spaceball, is used for 3D navigation with six-degree-of-freedom control, while the right hand, holding a mouse, is used to operate two-dimensional widgets and to measure morphological features by picking points on the volume. A simple 2D user interface in a separate panel is used for specifying the transfer function mapping data values to opacity/color values [6]. This way it is possible to interactively extract from the original data the required morphological features.

The two most important interactive operations are virtual camera control and point picking:

- virtual camera control: based on our previous work on physically based dynamic camera control, we interpret input from the $3 \mathrm{D}$ input device as forces and torques applied to a virtual camera model $[25,24,10]$. A viscous friction force field proportional to volume opacity is used to avoid penetration in opaque areas. This way, the endoscopic camera is always confined in the interior of a cavity; 


\section{References}

[1] Auer, L. M., Auer, D., And Knoplioch, J. F. Virtual endoscopy for planning and simulation of minimally invasive neurosurgery. Lecture Notes in Computer Science 1205 (1997), 315-336.

[2] Bier, E. A., Stone, M. C., Pier, K., Buxton, W., And DeRose, T. Toolglass and Magic Lenses: The see-through interface. In Computer Graphics (SIGGRAPH '93 Proceedings) (Aug. 1993), J. T. Kajiya, Ed., vol. 27, pp. 73-80.

[3] Cabral, B., Cam, N., And Foran, J. Accelerated volume rendering and tomographic reconstruction using texture mapping hardware. In 1994 Symposium on Volume Visualization (Oct. 1994), A. Kaufman and W. Krueger, Eds., ACM SIGGRAPH, pp. 91-98. ISBN 0-89791-741-3.

[4] Cullip, T. J., and Neumann, U. Accelerating volume reconstruction with 3D texture hardware. Tech. Rep. TR93027, Department of Computer Science, University of North Carolina - Chapel Hill, May 11994.

[5] Danskin, J., And Hanrahan, P. Fast algorithms for volume ray tracing. 1992 Workshop on Volume Visualization (1992), 91-98.

[6] Drebin, R. A., Carpenter, L., And Hanrahan, P. Volume rendering. Computer Graphics 22, 4 (Aug. 1988), 65-74.

[7] Favero, C. D., Belloni, G. M., and Martegani, A. Artifacts in virtual endoscopy. In Proceedings Of The 15th Europacs Annual Meeting (September 1997), C. Bortolazzi and D. Caramella, Eds., pp. 79-82.

[8] Giachetti, A., And Petri, C. Medical image transmission on heterogeneous network. In Proceedings Of The 15th Europacs Annual Meeting (September 1997), C. Bortolazzi and D. Caramella, Eds., pp. 117-120.

[9] GuAn, S., AND Lipes, R. G. Innovative volume rendering using 3D texture mapping. In Image Capture, Formatting and Display, vol. 2164 of SPIE. 1994.

[10] Hong, L., Muraki, S., Kaufman, A., Bartz, D., And HE, T. Virtual voyage: Interactive navigation in the human colon. In SIGGRAPH 97 Conference Proceedings (Aug. 1997), T. Whitted, Ed., Annual Conference Series, ACM SIGGRAPH, Addison Wesley, pp. 27-34. ISBN 0-89791-896-7.

[11] KULICK, T. Building an opengl volume renderer. SGI Dev. News, 1996

[12] Lorensen, W., Jolesz, F., And Kikinis, R. The exploration of cross-sectional data with a virtual endoscope. In Interactive Technology and the New Medical Paradigm for Health Care, R. Satava and K. Morgan, Eds. IOS Press, 1995, pp. 221-230.

[13] Max, N. Optical models for direct volume rendering. IEEE Transactions on Visualization and Computer Graphics 1, 2 (June 1995), 99-108.

[14] Mazuryk, T., Schmalstieg, D., and Gervautz, M. Zoom rendering: Improving 3-D rendering performance with 2-D operations. Tech. Rep. CG, Institute of Computer Graphics, Vienna University of Technology, 1995.

[15] Montrym, J. S., Baum, D. R., Dignam, D. L., And MigdAL, C. J. Infinite Reality: a real-time graphics system. Computer Graphics 31, 3A (Aug. 1997), 293-302.
[16] Neri, E., Caramella, D., Et al. Segmentation artifacts in spiral CT-angioscopy of the abdominal aorta. In Proceedings Of The 15th Europacs Annual Meeting (September 1997), C. Bortolazzi and D. Caramella, Eds., pp. 79-82.

[17] Ney, D. R., Fishman, E. K., Magid, D., And Drebin, R. A. Volumetric rendering of computed tomography data: Principles and techniques. IEEE Computer Graphics and Applications 10, 2 (Mar. 1990), 24-32.

[18] Prokop, M., Shin, H. O., Schanz, A., And SchaeferPROKOP, C. M. Use of maximum intensity projections in CT angiography: A basic review. Radiographics 17 (1997), 433-451.

[19] Rubin, G., Beaulieu, C., Argiro, V., Ringl, H., FEller, A. N. . J., DAKe, M., JeFFEY, R., AND NAPEL, $\mathrm{S}$. Perspective volume rendering of $\mathrm{CT}$ and MR images. $R a-$ diology 199 (1996), 321-330.

[20] SABELLA, P. A rendering algorithm for visualizing 3D scalar fields. Computer Graphics 22, 4 (Aug. 1988), 51-58.

[21] Serra, L., Hern, N., Choon, C. B., and Poston, T. Interactive vessel tracing in volume data. In Proceedings of the 1997 Symposium on Interactive 3D Graphics (Apr. 1997).

[22] Shahidi, R., Argiro, V., Napel, S., And Gray, L. Assessment of several virtual endoscopy techniques using computed tomography and perspective volume rendering. Lecture Notes in Computer Science 1131 (1996), 521-528.

[23] Summers, R. M. Navigational aids for real-time virtual bronchoscopy. AJR 168 (May 1997), 1165-1170.

[24] Turner, R., Balaguer, J.-F., Gobbetti, E., And THALMANN, D. Interactive scene walkthrough using a physically-based virtual camera. In CAAD Futures '91, Conference Proceedings (1991), G. N. Schmitt, Ed., Vieweg, pp. 511-520.

[25] Turner, R., Balaguer, J.-F., Gobbetti, E., And Thalmann, D. Physically-based interactive camera motion using 3D input devices. In Scientific Visualization of Physical Phenomena: Proceedings of CG International Tokyo (1991), N. M. Patrikalakis, Ed., Springer, pp. 135-145.

[26] Upson, C., And KeEler, M. V-BUFFER: Visible volume rendering. Computer Graphics 22, 4 (Aug. 1988), 59-64.

[27] VAn Gelder, A., AND Kim, K. Direct volume rendering with shading via three-dimensional textures. In 1996 Volume Visualization Symposium (Oct. 1996), IEEE, pp. 23-30. ISBN 0-89791-741-3

[28] WAShIngton UniVersity. MIR CTN documentation Version 2.8.3.

[29] You, S., Hong, L., Wan, M., Junyaprasert, K., KaufMAN, A., Muraki, S., ZhOU, Y., WaX, M., AND LiAnG, $Z$. Interactive volume rendering for virtual colonoscopy. In IEEE Visualization '97 (Nov. 1997), R. Yagel and H. Hagen, Eds., IEEE, pp. 343-346. 


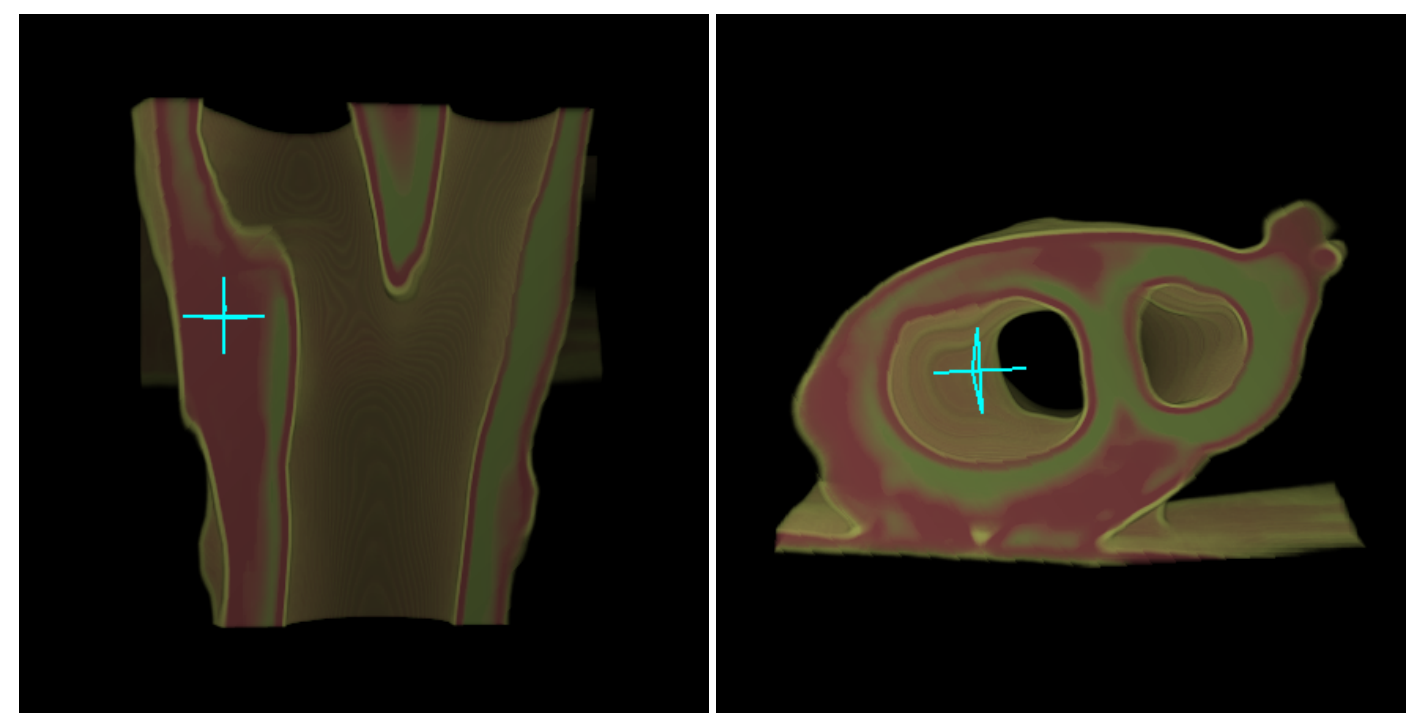

Figure 3: Cutting through an autoptic specimen of a human right carotid artery (256x256x32 CT dataset, 12 bit resolution, depth shading, Drebin classification). The left image shows the stenosis at the level of the carotid bifurcation, while the right one shows the diameter of the vessel seen from above. Cutting planes are positioned so as to identify interesting examination areas. The cursor indicates the region where plaque has formed.

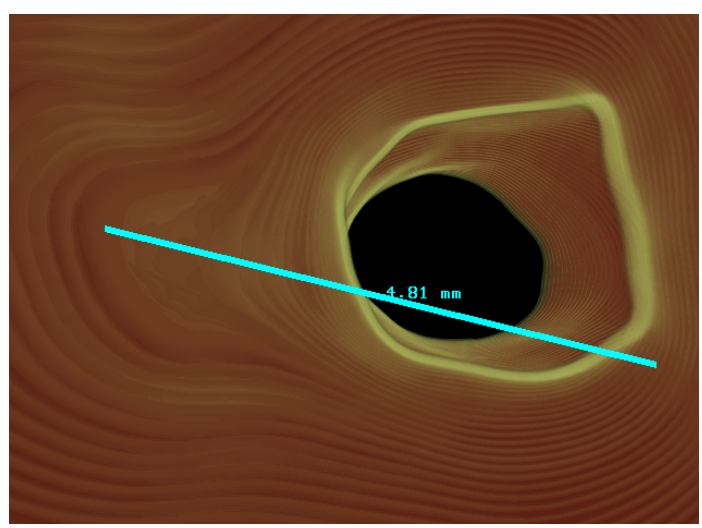

Figure 4: Endoscopic view of a stenosis area inside a human right carotid artery (256x256x32 CT dataset, 12 bit resolution, depth shading, Drebin classification). The surgeon has identified the stenosis area and interactively measures the variation in lumen of the vessel. The ruler is interactively dragged during examination, and the endpoints automatically follow the interior surface of the vessel. Here, the ruler is placed before the stenosis, measuring the lumen of the vessel when healthy.

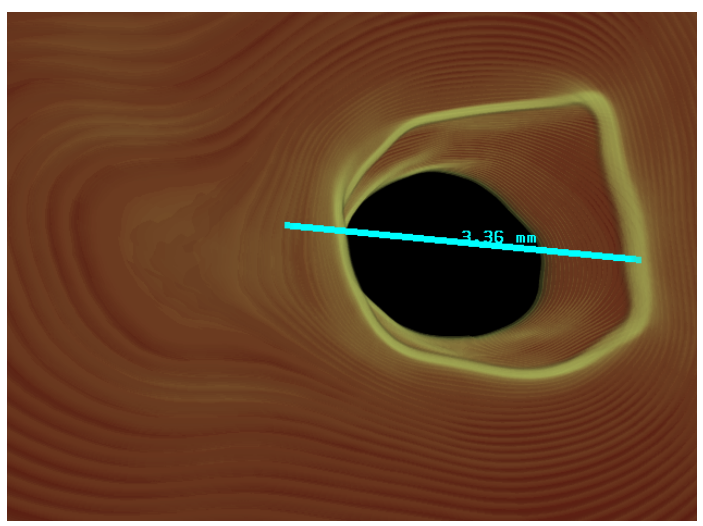

Figure 5: Endoscopic view of a stenosis area inside a human right carotid artery (256x256x32 CT dataset, 12 bit resolution, depth shading, Drebin classification). The surgeon has identified the stenosis area and interactively measures the variation in lumen of the vessel. The ruler is interactively dragged during examination, and the endpoints automatically follow the interior surface of the vessel. Here, the ruler shows clearly the actual luminal diameter in the stenosis area. 